\title{
The distribution of 4-full numbers
}

\author{
by \\ HONG-QUAN LIU (Harbin)
}

1. Introduction. A positive integer $n$ is called 4-full whenever $p \mid n$ implies that $p^{4} \mid n$, where $p$ denotes a prime number. Let $Q_{4}(x)$ be the number of 4-full numbers not exceeding $x$, for $x$ sufficiently large. The problem of finding an asymptotic formula for $Q_{4}(x)$ with a good error term has a long and distinguished history, beginning with a famous paper of Erdôs and Szekeres [3]. Elementary (Abel summation, Euler-Maclaurin summation), analytic (Perron formula, residue theorem), and exponential sum methods have subsequently been used to attack the problem. Let

$$
F(s)=\sum_{n=1}^{\infty} \frac{\chi(n)}{n^{s}} \quad \text { for } \operatorname{Re}(s)>1,
$$

where $\chi(n)$ is the character function of 4 -full integers; then

$$
F(s)=\frac{\zeta(4 s) \zeta(5 s) \zeta(6 s) \zeta(7 s)}{\zeta(10 s)} \sum_{n=1}^{\infty} \frac{c(n)}{n^{s}},
$$

the Dirichlet series $\sum c(n) n^{-s}$ is absolutely convergent for $\operatorname{Re}(s)>1 / 11$, so that by the residue theorem we can write

$$
Q_{4}(x)=\sum_{4 \leq i \leq 7} W_{i} x^{1 / i}+\Delta(x),
$$

with $\Delta(x)$ an error term. Let

$$
\lambda=\inf \left\{\varrho: \Delta(x) \ll x^{\varrho}\right\} .
$$

The following list of upper bounds of $\lambda$ can be found in the literature:

$1 / 5=0.2$, Erdős and Szekeres [3] (1935),

$1 / 6=0.1666 \ldots$, Bateman and Grosswald [2] (1958),

$169 / 1360=0.1242 \ldots$, Krätzel [7] (1972),

$257 / 2072=0.1240 \ldots$, Ivić [4] (1978),

$3187 / 25852=0.1232 \ldots$, Ivić [5] (1981),

$3091 / 25981=0.1189 \ldots$, Ivić and Shiu [6] (1982), 
$5 / 44=0.1136 \ldots$, Krätzel [8] (1983),

$21 / 187=0.1122 \ldots$, Krätzel [10] (1989);

in particular, the last result of Krätzel was obtained by using the threedimensional lattice point results of his paper [9]. Professor Krätzel informed the author in June 1993 that Dr. Menzer (Jena) already got a further improvement on his result.

The purpose of this paper is to give a better upper bound for $\lambda$. We will show the following

Theorem 1. $\lambda \leq 6 / 59=0.1016 \ldots$

Our result is near but still falls short of the expected bound, namely, $\lambda \leq 0.1$. In light of the argument involved in Krätzel [10], it suffices to deduce the following

Theorem 2. For any $\varepsilon>0$,

$$
\sum_{n_{1}^{4} n_{2}^{5} n_{3}^{6} n_{4}^{7} \leq x} 1=A x^{1 / 4}+B x^{1 / 5}+C x^{1 / 6}+D x^{1 / 7}+O\left(x^{6 / 59+\varepsilon}\right)
$$

with some absolute constants $A, B, C$ and $D$.

Following the approach of Krätzel, Theorem 2 can be reduced to 4-dimensional exponential sums, which can be estimated by a combination of Kolesnik's method and a refined version of the Bombieri-Fouvry-Iwaniec method (cf. [11], [12]).

The author is grateful to Professor Ekkehard Krätzel for providing a lot of reprints of his papers on divisors problems, without which the present work would not exist.

2. Proof of Theorem 2 (reduction). From (4), (5) and (7) of [10],

$$
\sum_{n_{1}^{4} n_{2}^{5} n_{3}^{6} n_{4}^{7} \leq x} 1=A x^{1 / 4}+B x^{1 / 5}+C x^{1 / 6}+D x^{1 / 7}+E(x)
$$

where

$$
E(x)=-\sum_{(a, b, c, d)} S(a, b, c, d ; x)+O\left(x^{1 / 11}\right),
$$

$(a, b, c, d)$ runs through all permutations of $(4,5,6,7)$, and

$$
S(a, b, c, d ; x)=\sum_{1} \psi\left(\left(\frac{x}{n_{1}^{a} n_{2}^{b} n_{3}^{c}}\right)^{1 / d}\right),
$$

$\psi(t)=t-[t]-\frac{1}{2}([t]$ is the integral part of $t)$, with $\sum_{1}$ denoting summation over all lattice points $\left(n_{1}, n_{2}, n_{3}\right)$ with

$$
n_{1}^{a} n_{2}^{b} n_{3}^{c+d} \leq x, \quad 1 \leq n_{1}(\leq) n_{2} \leq n_{3} ;
$$


here $\left.n_{1}(\leq) n_{2}\right)$ means $n_{1} \leq n_{2}$ if $(a, b)=\left(a_{i}, a_{j}\right)$ for $i<j$, and $n_{1}<$ $n_{2}$ otherwise, and we have set $(4,5,6,7)=\left(a_{1}, a_{2}, a_{3}, a_{4}\right)$. We can write $S(a, b, c, d ; x)$ as $O\left((\ln x)^{3}\right)$ subsums of the type $S(a, b, c, d ; \boldsymbol{N})$, together with a permissible error, where $\boldsymbol{N}=\left(N_{1}, N_{2}, N_{3}\right), N_{v}$ 's are positive integers, and

$$
S(a, b, c, d ; \boldsymbol{N})=\sum_{2} \psi\left(\left(\frac{x}{n_{1}^{a} n_{2}^{b} n_{3}^{c}}\right)^{1 / d}\right)
$$

with $\sum_{2}$ denoting summation over lattice points $\left(n_{1}, n_{2}, n_{3}\right)$ with

(0) $n_{1}^{a} n_{2}^{b} n_{3}^{c+d} \leq x, \quad 1 \leq n_{1}(\leq) n_{2} \leq n_{3}, \quad N_{v} \leq n_{v}<2 N_{v} \quad(v=1,2,3)$.

By means of the Fourier series treatment of the function $\psi(t)$ (cf. (18) and (19) of [11]), for a parameter $K \in\left[10, x^{1 / 2}\right]$ and some number $H \in\left[1, K^{2}\right]$ ( $H$ depends on $K$ ), we have the estimate

$$
\begin{aligned}
x^{-\varepsilon} S(a, b, c, d ; \boldsymbol{N}) \ll & N_{1} N_{2} N_{3} K^{-1} \\
& +\min (1, K / H)(\Phi(H ; \boldsymbol{N})+\Psi(H ; \boldsymbol{N})),
\end{aligned}
$$

where

$$
\begin{gathered}
\Phi(H ; \boldsymbol{N})=H^{-1} \sum_{h \sim H}\left|\sum_{3} e\left(f\left(h, n_{1}, n_{2}, n_{3}\right)\right)\right|, \\
f\left(h, n_{1}, n_{2}, n_{3}\right)=h\left(\frac{x}{n_{1}^{a} n_{2}^{b} n_{3}^{c}}\right)^{1 / d}
\end{gathered}
$$

with $\sum_{3}$ denoting summation over lattice points $\left(n_{1}, n_{2}, n_{3}\right)$ with

(*) $n_{1}^{a} n_{2}^{b} n_{3}^{c+d} \leq x, \quad 1 \leq n_{1}<n_{2} \leq n_{3}, \quad N_{v} \leq n_{v}<2 N_{v} \quad(v=1,2,3)$,

and

$$
\begin{gathered}
\Psi(H ; \boldsymbol{N})=H^{-1} \sum_{h \sim H}\left|\sum_{4} e\left(f_{1}\left(h, n_{2}, n_{3}\right)\right)\right|, \\
f_{1}\left(h, n_{2}, n_{3}\right)=h\left(x n_{2}^{-a-b} n_{3}^{-c}\right)^{1 / d}
\end{gathered}
$$

with $\sum_{4}$ denoting summation over lattice points $\left(n_{2}, n_{3}\right)$ with

(\#) $\quad n_{2}^{a+b} n_{3}^{c+d} \leq x, \quad 1 \leq n_{2} \leq n_{3}, \quad N_{v} \leq n_{v}<2 N_{v} \quad(v=2,3)$

(that is, (\#) is obtained from (0) by taking $n_{1}=n_{2}$ ).

Throughout this paper we use the notations $r \sim R$ and $r \cong R$ to mean $1 \leq r / R<2$ and $C_{1} \leq r / R \leq C_{2}$, respectively; $C_{i}(i=1,2,3, \ldots)$ will be some absolute constants. As usual, $e(\xi)=\exp (2 \pi i \xi)$ for a real number $\xi$. 
As the contribution of $\Psi(H ; \boldsymbol{N})$ is always negligible when compared with that of $\Phi(H ; \boldsymbol{N})$, we will omit $\Psi(H ; \boldsymbol{N})$ from our argument throughout. For convenience we can assume that $x=\sqrt{5} \cdot Z$, where $Z$ is an integer, that is, $x$ is a quadratic irrational (otherwise we can replace $x$ by $5^{1 / 2}\left[x 5^{-1 / 2}\right]$ and add a permissible error in (1)). To deal with $\Phi(H ; \boldsymbol{N})$ we first transform summation over $n_{3}$ to summation over $u$ via the following lemma.

Lemma 1. Let $f(x)$ and $g(x)$ be algebraic functions for $x \in[a, b]$, satisfying

$$
\begin{gathered}
\left|f^{\prime \prime}(x)\right| \cong R^{-1}, \quad f^{\prime \prime \prime}(x) \ll(R U)^{-1} \\
|g(x)| \leq H, \quad g^{\prime}(x) \ll H U_{1}^{-1}, \quad U, U_{1} \geq 1 .
\end{gathered}
$$

Then

$$
\begin{aligned}
\sum_{a \leq n \leq b} g(n) e(f(n)) & \\
= & \sum_{\alpha \leq u \leq \beta} b_{u} \frac{g(n(u))}{\sqrt{f^{\prime \prime}(n(u))}} e(f(n(u))-u n(u)+1 / 8) \\
& +O\left(H \ln (\beta-\alpha+2)+H(b-a+R)\left(U^{-1}+U_{1}^{-1}\right)\right) \\
& +O\left(H \min \left(R^{1 / 2}, \max \left(\frac{1}{\langle\alpha\rangle}, \frac{1}{\langle\beta\rangle}\right)\right)\right),
\end{aligned}
$$

where $[\alpha, \beta]$ is the image of $[a, b]$ under the mapping $y=f^{\prime}(x), n(u)$ is determined by the equation $f^{\prime}(n(u))=u$, and

$$
b_{u}= \begin{cases}1 & \text { for } \alpha<u<\beta, \\ 1 / 2 & \text { for } u=\alpha=\text { integer or } u=\beta=\text { integer } ;\end{cases}
$$

the function $\langle x\rangle$ is defined as follows:

$$
\langle x\rangle= \begin{cases}\|x\| & \text { if } x \text { is not an integer }, \\ \beta-\alpha & \text { otherwise, }\end{cases}
$$

where $\|x\|=\min _{n \in \mathbb{Z}}|x-n| ;$ and $\sqrt{f^{\prime \prime}}>0$ if $f^{\prime \prime}>0$, and $\sqrt{f^{\prime \prime}}=i \sqrt{\left|f^{\prime \prime}\right|}$ if $f^{\prime \prime}<0$.

Proof. This is Lemma 1.4 of [13].

Now put

$$
\begin{gathered}
X=x n_{1}^{-a} \\
M_{1}=\max \left(N_{3}, n_{2}\right), \quad M_{2}=\min \left(\left(X n_{2}^{-b}\right)^{1 /(c+d)}, 2 N_{3}\right), \\
U_{1}=\frac{h c}{d}\left(X n_{2}^{-b} M_{2}^{-c-d}\right)^{1 / d}, \quad U_{2}=\frac{h c}{d}\left(X n_{2}^{-b} M_{1}^{-c-d}\right)^{1 / d} .
\end{gathered}
$$


Lemma 1 yields

(3)

$$
\begin{aligned}
& \sum_{M_{1} \leq n_{3} \leq M_{2}} e(g) \\
& =\sum_{U_{1}<u<U_{2}} C_{1}\left(X^{-1} h^{-d} n_{2}^{b} u^{2 d+c}\right)^{1 /(2(d+c))} e(g) \\
& \quad+O\left(N_{3}(H F)^{-1}+\ln x+\min \left(\left(N_{3}^{2} H^{-1} F^{-1}\right)^{1 / 2}, \frac{1}{U_{2}-h c / d}\right)\right) \\
& \quad+O\left(\sum_{1 \leq i \leq 2} \min \left(\left(N_{3}^{2} H^{-1} F^{-1}\right)^{1 / 2}, \frac{1}{\left\|T\left(n_{2}, X_{i}\right)\right\|}\right)\right) \\
& \quad+R\left(h, n_{1}, n_{2}\right),
\end{aligned}
$$

where

$$
\begin{gathered}
g=C_{2}\left(X h^{d} u^{c} n_{2}^{-b}\right)^{1 /(c+d)}, \quad X_{1}=\max \left(n_{2}, N_{3}\right), \quad X_{2}=2 N_{3}, \\
F=\left(X N_{3}^{-c} N_{2}^{-b}\right)^{1 / d}, \\
R\left(h, n_{1}, n_{2}\right)=\left\{\begin{array}{c}
\frac{1}{2} C_{1}\left(X^{-1} h^{-d} n_{2}^{b} U_{1}^{2 d+c}\right)^{-1 /(2(c+d))} e(g) \\
\text { if } M_{2}=\left(X n_{2}^{-b}\right)^{1 /(d+c)} \text { and } U_{1} \text { integer, } \\
0 \quad \text { otherwise, } \\
T\left(n_{2}, w\right)=\frac{h c}{d}\left(X n_{2}^{-b} w^{-d-c}\right)^{1 / d} .
\end{array}\right.
\end{gathered}
$$

We find that (as $F \gg N_{3}$ )

$$
\sum_{n_{1}} \sum_{n_{2}} \min \left(\left(N_{3}^{2} H^{-1} F^{-1}\right)^{1 / 2}, \frac{1}{U_{2}-h c / d}\right) \ll x^{1 / 11} .
$$

Let $G=\left(x N_{1}^{-a} N_{2}^{-b} N_{3}^{-c}\right)^{1 / d}(\cong F)$. By Hilfssatz 4 of [9] we get

(5)

$$
\begin{aligned}
\sum_{n_{1}} \sum_{n_{2}} & \min \left(\left(N_{3}^{2} H^{-1} F^{-1}\right)^{1 / 2}, \frac{1}{\left\|T\left(n_{2}, X_{i}\right)\right\|}\right) \\
& \ll N_{1}\left(1+H G N_{3}^{-1}\right)\left(\left(N_{3}^{2} H^{-1} G^{-1}\right)^{1 / 2}+H^{-1} G^{-1} N_{2} N_{3}\right) \ln x \\
& \ll N_{1}(H G)^{1 / 2} \ln x+x^{1 / 11} .
\end{aligned}
$$

Finally, an application of the exponent pair $(1 / 6,4 / 6)$ gives

(6) $\quad \sum_{n_{1}} \sum_{n_{2}} R\left(h, n_{1}, n_{2}\right)$

$$
\begin{aligned}
& \ll N_{1}\left(N_{3}^{2}(H G)^{-1}\right)^{1 / 2}\left(N_{2}^{4 / 6}\left(G H N_{2}^{-1}\right)^{1 / 6}+N_{2} H^{-1} G^{-1}\right) \\
& \ll N_{1} N_{2}^{1 / 2} N_{3} G^{-1 / 3}+N_{1} N_{2} N_{3} G^{-3 / 2} \ll N_{1} N_{2}^{1 / 2} N_{3}^{2 / 3}+N_{1} N_{2} \\
& \ll\left(N_{1}^{7} N_{2}^{6} N_{3}^{9}\right)^{13 / 132}+N_{1} N_{2} \ll x^{13 / 132} .
\end{aligned}
$$


From (2)-(6) we get

$$
\begin{gathered}
\Phi(H ; \boldsymbol{N}) \ll H^{-1}\left(N_{3}^{2} H^{-1} G^{-1}\right)^{1 / 2} \sum_{h \sim H}\left|\sum_{5} g_{1}\left(n_{1}\right) g_{2}\left(n_{2}\right) g_{3}(u) e(g)\right| \\
+N_{1}(H G)^{1 / 2} \ln x+x^{13 / 132}
\end{gathered}
$$

where $\sum_{5}$ denotes summation over lattice points $\left(n_{1}, n_{2}, u\right)$ with

$$
1 \leq n_{1}<n_{2}, \quad N_{v} \leq n_{v}<2 N_{v} \quad(v=1,2), \quad U_{1}<u<U_{2},
$$

and $g_{i}(\cdot)(i=1,2,3)$ are monomials with $\left|g_{i}(\cdot)\right| \leq 1$.

\section{Three estimates for $S(a, b, c, d ; \boldsymbol{N})$}

Lemma 2. Let $f(x, y)=A x^{\alpha} y^{\beta}, g(x, y)=B x^{\gamma} y^{\delta}$, where $\alpha, \beta, \gamma$ and $\delta$ are rationals with $\alpha \beta(\alpha+\beta-1)(\alpha+\beta-2) \neq 0, A>0$, and suppose that $g(x, y) \cong G$ holds for $(x, y)$ with $x \sim X$ and $y \sim Y$. Moreover, suppose that $D \subset\{(x, y) \mid x \sim X, y \sim Y\}, D$ is embraced by $O(1)$ algebraic curves, and $X \gg Y$. Let $N=X Y, F=A X^{\alpha} Y^{\beta}$. Then

$$
\begin{aligned}
\sum_{(x, y) \in D} g(x, y) e(f(x, y)) \ll & (N+A N)^{\varepsilon} G\left(\sqrt[6]{F^{2} N^{3}}+N^{5 / 6}\right. \\
& \left.+\sqrt[8]{N^{8} F^{-1} X^{-1}}+N F^{-1 / 4}+N Y^{-1 / 2}\right) .
\end{aligned}
$$

Proof. This is a "weighted" version of Lemma 9 of [11], and can be obtained similarly by Kolesnik's original method.

Applying Lemma 2 to the variables $n_{2}$ and $u$ of the multiple sum of (7), we get

$$
\begin{aligned}
x^{-\varepsilon} \Phi(H ; \boldsymbol{N}) \ll & \sqrt[6]{(H G)^{2} N_{1}^{6} N_{2}^{3} N_{3}^{3}}+\sqrt[8]{(H G)^{3} N_{1}^{8} N_{2}^{7}} \\
& +(H G)^{1 / 2} N_{1} N_{2}^{1 / 2}+N_{1} N_{2} N_{3}^{1 / 2}+x^{\varphi},
\end{aligned}
$$

where $\varphi=13 / 132$; thus from (1) we obtain

$$
\begin{aligned}
x^{-2 \varepsilon} S(a, b, c, d ; \boldsymbol{N}) \ll & N_{1} N_{2} N_{3} K^{-1}+\sqrt[6]{(K G)^{2} N_{1}^{6} N_{2}^{3} N_{3}^{3}} \\
& +\sqrt[8]{(K G)^{3} N_{1}^{8} N_{2}^{7}}+(K G)^{1 / 2} N_{1} N_{2}^{1 / 2} \\
& +N_{1} N_{2} N_{3}^{1 / 2}+x^{\varphi} .
\end{aligned}
$$

To choose $K$ optimally we need the following

Lemma 3. Let $M, N, u_{m}, v_{n}, A_{m}, B_{n}$ be positive $(1 \leq n \leq N, 1 \leq m$ $\leq M)$, and $Q_{1}$ and $Q_{2}$ be given non-negative numbers with $Q_{1}<Q_{2}$. Then 
there is a number $Q$ such that $Q_{1} \leq Q \leq Q_{2}$ and

$$
\begin{aligned}
\sum_{1 \leq m \leq M} A_{m} Q^{u_{m}}+\sum_{1 \leq n \leq N} B_{n} Q^{-v_{n}} \ll & \sum_{1 \leq n \leq N} \sum_{1 \leq m \leq M}\left(A_{m}^{v_{n}} B_{n}^{u_{m}}\right)^{1 /\left(u_{m}+v_{n}\right)} \\
& +\sum_{1 \leq m \leq M} A_{m} Q_{1}^{u_{m}}+\sum_{1 \leq n \leq N} B_{n} Q_{2}^{-v_{n}} .
\end{aligned}
$$

Proof. This is Lemma 2 of [11].

By Lemma 3 we can choose a $K \in\left[0, x^{1 / 2}\right]$ optimally in (8) so that

$$
\begin{aligned}
x^{-2 \varepsilon} S(a, b, c, d ; \boldsymbol{N}) \ll & \sqrt[8]{G^{2} N_{1}^{8} N_{2}^{5} N_{3}^{5}}+\sqrt[11]{G^{3} N_{1}^{11} N_{2}^{10} N_{3}^{3}} \\
& +\sqrt[3]{G N_{1}^{3} N_{2}^{2} N_{3}}+N_{1} N_{2} N_{3}^{1 / 2}+x^{\varphi} .
\end{aligned}
$$

This is our first estimate. To get the second, we apply the next

Lemma 4. Let $H \geq 1, X \geq 1, Y \geq 1000, \alpha, \beta$ and $\gamma$ be real numbers with $\alpha \gamma(\gamma-1)(\beta-1) \neq 0$, and let $A>C(\alpha, \beta, \gamma)>0$ and $f(h, x, y)=A h^{\alpha} x^{\beta} y^{\gamma}$. Define

$$
S(H, X, Y)=\sum_{(h, x, y) \in D} C_{1}(h, x) C_{2}(y) e(f(h, x, y)),
$$

where $D$ is a region contained in the rectangle $\{(h, x, y) \mid h \sim H, x \sim X$, $y \sim Y\}$ such that for any fixed pair $\left(h_{0}, x_{0}\right)$, the intersection $D \cap\left\{\left(h_{0}, x_{0}, y\right) \mid\right.$ $y \sim Y\}$ has at most $O(1)$ segments. Also, suppose that $\left|C_{1}(h, x)\right| \leq 1$, $\left|C_{2}(y)\right| \leq 1$ and $F=A H^{\alpha} X^{\beta} Y^{\gamma} \gg Y$. Then, for $L=\ln ((A+1) H X Y+2)$ and $M=\max \left(1, F Y^{-2}\right)$,

$$
\begin{aligned}
L^{-3} S(H, X, Y) \ll & \sqrt[22]{(H X)^{19} Y^{13} F^{3}}+H X Y^{5 / 8}\left(1+Y^{7} F^{-4}\right)^{1 / 16} \\
& +\sqrt[32]{(H X)^{29} Y^{28} F^{-2} M^{5}}+\sqrt[4]{(H X)^{3} Y^{4} M}
\end{aligned}
$$

Proof. This is Theorem 3 of [12].

We apply Lemma 4 to the triple $\left(h, u, n_{2}\right)$ of $(7)$, with the choice $(h, x, y)$ $=\left(h, u, n_{2}\right)$, and we estimate the sum over $n_{1}$ trivially in (7), thus obtaining (note that $u \cong H G N_{3}^{-1}$ ):

$$
\begin{aligned}
x^{-\varepsilon} \Phi(H ; \boldsymbol{N}) \ll & \sqrt[22]{H^{8} G^{11} N_{1}^{22} N_{2}^{13} N_{3}^{3}}+(H G)^{1 / 2} N_{1} N_{2}^{5 / 8} \\
& +\sqrt[16]{(H G)^{4} N_{1}^{16} N_{2}^{17}}+\sqrt[32]{H^{8} G^{11} N_{1}^{32} N_{2}^{28} N_{3}^{3}} \\
& +\sqrt[32]{H^{13} G^{16} N_{1}^{32} N_{2}^{18} N_{3}^{3}}+\sqrt[4]{G N_{1}^{4} N_{2}^{4} N_{3}} \\
& +\sqrt[4]{(H G)^{2} N_{1}^{4} N_{2}^{2}}+x^{\varphi} .
\end{aligned}
$$

From (1) and (10) we find that 
(11)

$$
\begin{aligned}
x^{-2 \varepsilon} S(a, b, c, d ; \boldsymbol{N}) \ll & N_{1} N_{2} N_{3} K^{-1} \\
& +\sqrt[22]{K^{8} G^{11} N_{1}^{22} N_{2}^{13} N_{3}^{3}}+(K G)^{1 / 2} N_{1} N_{2}^{5 / 8} \\
& +\sqrt[16]{(K G)^{4} N_{1}^{16} N_{2}^{17}}+\sqrt[32]{K^{8} G^{11} N_{1}^{32} N_{2}^{28} N_{3}^{3}} \\
& +\sqrt[32]{K^{13} G^{16} N_{1}^{32} N_{2}^{18} N_{3}^{3}}+\sqrt[4]{G N_{1}^{4} N_{2}^{4} N_{3}} \\
& +\sqrt[4]{(K G)^{2} N_{1}^{4} N_{2}^{2}}+x^{\varphi}
\end{aligned}
$$

Choosing $K \in\left[0, x^{1 / 2}\right]$ optimally via Lemma 3 , we obtain

$$
\begin{aligned}
x^{-2 \varepsilon} S(a, b, c, d ; \boldsymbol{N}) \ll & \sqrt[30]{G^{11} N_{1}^{30} N_{2}^{21} N_{3}^{11}}+\sqrt[24]{\left(G N_{3}\right)^{8} N_{2}^{18} N_{1}^{24}} \\
& +\sqrt[20]{\left(G N_{3}\right)^{4} N_{2}^{21} N_{1}^{20}}+\sqrt[40]{\left(G N_{3}\right)^{11} N_{2}^{36} N_{1}^{40}} \\
& +\sqrt[45]{\left(G N_{3}\right)^{16} N_{2}^{31} N_{1}^{45}}+\sqrt[5]{\left(G N_{3}\right)^{2} N_{2}^{3} N_{1}^{5}} \\
& +\sqrt[4]{G N_{3} N_{2}^{4} N_{1}^{4}}+x^{\varphi}
\end{aligned}
$$

This is the second estimate. To deduce the third, we first relax the severe constraint $n_{1}<n_{2}$ and $U_{1}<u<U_{2}$ by the next familiar lemma.

Lemma 5. Let $T, \alpha$ and $\beta$ be real, $T>0, \beta>0$. Then

$$
\frac{1}{\pi} \int_{-T}^{T} e^{i t \alpha} \frac{\sin t \beta}{t} d t= \begin{cases}1+O(1 /(T(\beta-|\alpha|))) & \text { if }|\alpha| \leq \beta \\ O(1 /(T(|\alpha|-\beta))) & \text { if }|\alpha|>\beta .\end{cases}
$$

We can apply Lemma 5 as follows. For instance, we want to remove the inequality

$$
u>U_{1}=\frac{h c}{d}\left(x n_{1}^{-a} n_{2}^{-b}\left(2 N_{3}\right)^{-c-d}\right)^{1 / d}
$$

when $M_{2}=2 N_{3}$; then we choose $T=x^{1000}, \alpha=\ln \left(x(h c)^{d}\right)$ and $\beta=$ $\ln \left((u d)^{d} n_{1}^{a} n_{2}^{b}\left(2 N_{3}\right)^{c+d}\right)$ in Lemma 5 . As $x=\sqrt{5} Z$ with $Z$ an integer, the contribution of $O\left(T(|| \alpha|-\beta|)^{-1}\right)$ of Lemma 5 can be estimated satisfactorily by using the fact that $|x-p / q| \gg q^{-2} x^{-1}$ for all rationals $p / q$ with $q>0$ (which is implied by a special case of Liouville's theorem, cf. $\S 5$ of Chapter 6 in Baker [1]). In this fashion we can remove all the relationships between the lattice points $\left(h, n_{2}\right)$ and $\left(n_{1}, u\right)$ consecutively. Thus from $(7)$ we get

$$
\begin{aligned}
& x^{-\varepsilon} \Phi(H ; \boldsymbol{N}) \\
\ll & \left(H^{-3} G^{-1} N_{3}^{2}\right)^{1 / 2} \sum_{h \sim H} \sum_{n_{2} \sim N_{2}}\left|\sum_{\left(n_{1}, u\right) \in D_{1}} C\left(n_{1}, u\right) e\left(C_{2}\left(x h^{d} u^{c} n_{1}^{-a} n_{2}^{-b}\right)^{\delta}\right)\right| \\
+ & N_{1}(H G)^{1 / 2}+x^{\varphi},
\end{aligned}
$$

where, for brevity, $\delta=1 /(c+d)$, and $D_{1}$ is a region contained in $\left\{\left(n_{1}, u\right) \mid\right.$ $\left.n_{1} \sim N_{1}, u \cong U=H G N_{3}^{-1}\right\}$, independent of $h$ and $n_{2} ;\left|C\left(n_{1}, u\right)\right| \leq 1$. By 
Lemma 4 of [11] we get from (13) the estimate

$$
x^{-2 \varepsilon} \Phi(H ; \boldsymbol{N})^{2} \ll H^{-2} N_{3}^{2} B_{1} B_{2}+H G N_{1}^{2}+x^{2 \varphi},
$$

where $B_{1}$ is the number of lattice points $\left(h, n_{2}, \widetilde{h}, \widetilde{n}_{2}\right)$ such that

$$
h, \widetilde{h} \sim H, \quad n_{2}, \widetilde{n}_{2} \sim N_{2}, \quad\left|\left(h^{d} n_{2}^{-b}\right)^{1 /(c+d)}-\left(\widetilde{h}^{d} \widetilde{n}_{2}^{-b}\right)^{1 /(c+d)}\right| \ll \Delta R_{1},
$$

with $\Delta=(H G)^{-1}$ and $R_{1}=\left(H^{d} N_{2}^{-b}\right)^{1 /(c+d)}$, and where $B_{2}$ is the number of lattice points $\left(n_{1}, u, \widetilde{n}_{1}, \widetilde{u}\right)$ such that

$$
\begin{gathered}
n_{1}, \widetilde{n}_{1} \sim N_{1}, \quad u, \widetilde{u} \cong H G N_{3}^{-1}, \\
\left|\left(u^{c} n_{1}^{-a}\right)^{1 /(c+d)}-\left(\widetilde{u}^{c} \widetilde{n}_{1}^{-a}\right)^{1 /(c+d)}\right| \ll \Delta R_{2},
\end{gathered}
$$

with $R_{2}=\left(\left(G H N_{3}^{-1}\right)^{c} N_{1}^{-a}\right)^{1 /(c+d)}$. By Lemma 5 of [11],

(15) $\quad B_{1} \ll\left(H N_{2}+H N_{2} N_{2} G^{-1}\right)(\ln x)^{2} \ll H N_{2} \ln ^{2} x$,

(16) $\quad B_{2} \ll\left(N_{1} H G N_{3}^{-1}+H G N_{1}^{2} N_{3}^{-2}\right)(\ln x)^{2} \ll N_{1} H G N_{3}^{-1} \ln ^{2} x$.

From (14)-(16) we get

$$
x^{-2 \varepsilon} \Phi(H ; \boldsymbol{N}) \ll\left(G N_{1} N_{2} N_{3}\right)^{1 / 2}+N_{1}(H G)^{1 / 2}+x^{\varphi},
$$

which, in conjunction with (1) and the choice $K=\left(G^{-1} N_{2}^{2} N_{3}^{3}\right)^{1 / 3}$, gives

$$
\begin{aligned}
x^{-3 \varepsilon} S(a, b, c, d ; \boldsymbol{N}) & \ll\left(G N_{1} N_{2} N_{3}\right)^{1 / 2}+\sqrt[3]{G N_{1}^{3} N_{2} N_{3}}+x^{\varphi} \\
& \ll\left(G N_{1} N_{2} N_{3}\right)^{1 / 2}+x^{\varphi} .
\end{aligned}
$$

This is our third estimate.

4. Proof of Theorem 2 (completion). Recall that $N_{1} \ll N_{2} \ll N_{3}$; thus

$$
\left(N_{3} \ll\right) G:=\left(x N_{1}^{-a} N_{2}^{-b} N_{3}^{-c}\right)^{1 / d} \ll\left(x N_{1}^{-7} N_{2}^{-6} N_{3}^{-5}\right)^{1 / 4}
$$

for any permutation $(a, b, c, d)$ of $(4,5,6,7)$. By our three estimates (9), (12), (17), and the fact (18), we have, with $\eta=4 \varepsilon$,

$$
\begin{aligned}
x^{-\eta} S(a, b, c, d ; \boldsymbol{N}) \ll & \sqrt[16]{x N_{1}^{9} N_{2}^{4} N_{3}^{5}}+\sqrt[44]{x^{3} N_{1}^{23} N_{2}^{22} N_{3}^{-3}} \\
& +\sqrt[12]{x N_{1}^{5} N_{2}^{2} N_{3}^{-1}}+N_{1} N_{2} N_{3}^{1 / 2}+x^{\varphi} \\
\ll & \sqrt[16]{x N_{1}^{9} N_{2}^{4} N_{3}^{5}}+\sqrt[44]{x^{3} N_{1}^{23} N_{2}^{19}} \\
& +\sqrt[12]{x N_{1}^{5} N_{2}}+N_{1} N_{2} N_{3}^{1 / 2}+x^{\varphi}
\end{aligned}
$$


(20)

$$
\begin{aligned}
x^{-\eta} S(a, b, c, d ; \boldsymbol{N}) \ll & \sqrt[120]{x^{11} N_{1}^{43} N_{2}^{18} N_{3}^{-11}}+\sqrt[24]{x^{2} N_{1}^{10} N_{2}^{6} N_{3}^{-2}} \\
& +\sqrt[20]{x N_{1}^{13} N_{2}^{15} N_{3}^{-1}}+\sqrt[160]{x^{11} N_{1}^{83} N_{2}^{78} N_{3}^{-11}} \\
& +\sqrt[45]{x^{4} N_{1}^{17} N_{2}^{7} N_{3}^{-4}}+\sqrt[10]{x N_{1}^{3} N_{3}^{-1}} \\
& +\sqrt[16]{x N_{1}^{9} N_{2}^{10} N_{3}^{-1}}+x^{\varphi}
\end{aligned}
$$

and

$$
x^{-\eta} S(a, b, c, d ; \boldsymbol{N}) \ll \sqrt[8]{x N_{1}^{-3} N_{2}^{-2} N_{3}^{-1}}+x^{\varphi} .
$$

It remains to deduce the required estimate from (19)-(21).

From (19) and (21) it is seen that

$$
x^{-\eta} S(a, b, c, d ; \boldsymbol{N}) \ll \sqrt[16]{x N_{1}^{9} N_{2}^{4} N_{3}^{5}}+E_{1}+E_{2}+E_{3}+x^{\varphi},
$$

where

$$
\begin{aligned}
& E_{1}=\min \left(\sqrt[44]{x^{3}\left(N_{1} N_{2}\right)^{21}}, \sqrt[8]{x\left(N_{1} N_{2}\right)^{-3}}\right) \leq x^{0.1} \\
& E_{2}=\min \left(\sqrt[12]{x\left(N_{1} N_{2}\right)^{3}}, \sqrt[8]{x\left(N_{1} N_{2}\right)^{-3}}\right) \leq x^{0.1} \\
& E_{3}=\min \left(N_{1} N_{2} N_{3}^{1 / 2}, \sqrt[8]{x N_{1}^{-3} N_{2}^{-2} N_{3}^{-1}}\right) \ll\left(x N_{1}^{-1}\right)^{0.1} \leq x^{0.1}
\end{aligned}
$$

Thus

$$
x^{-\eta} S(a, b, c, d ; \boldsymbol{N}) \ll \sqrt[16]{x N_{1}^{9} N_{2}^{4} N_{3}^{5}}+x^{0.1} .
$$

From (20) and (22) we obtain

$$
x^{-\eta} S(a, b, c, d ; \boldsymbol{N}) \ll \sum_{4 \leq i \leq 10} E_{i}+x^{0.1},
$$

where

(24) $\quad E_{4}=\min \left(\sqrt[10]{x N_{1}^{3} N_{3}^{-1}}, \sqrt[16]{x N_{1}^{9} N_{2}^{4} N_{3}^{5}}\right) \ll \sqrt[33]{x^{3} N_{1}^{12} N_{2}^{2}}$,

(25) $\quad E_{5}=\min \left(\sqrt[120]{x^{11} N_{1}^{43} N_{2}^{18} N_{3}^{-18}}, \sqrt[16]{x N_{1}^{9} N_{2}^{4} N_{3}^{5}}\right)$

$$
\ll \sqrt[888]{x^{73} N_{1}^{377} N_{2}^{162}}
$$

(26) $\quad E_{6}=\min \left(\sqrt[12]{x N_{1}^{5} N_{2}^{3} N_{3}^{-1}}, \sqrt[16]{x N_{1}^{9} N_{2}^{4} N_{3}^{5}}\right) \ll \sqrt[76]{x^{6} N_{1}^{34} N_{2}^{19}}$,

(27) $\quad E_{7}=\min \left(\sqrt[20]{x N_{1}^{13} N_{2}^{15} N_{3}^{-1}}, \sqrt[16]{x N_{1}^{9} N_{2}^{4} N_{3}^{5}}\right) \ll \sqrt[116]{x^{6} N_{1}^{74} N_{2}^{79}}$,

(28) $\quad E_{8}=\min \left(\sqrt[160]{x^{11} N_{1}^{83} N_{2}^{78} N_{3}^{-11}}, \sqrt[16]{x N_{1}^{9} N_{2}^{4} N_{3}^{5}}\right)$

$$
\ll \sqrt[488]{x^{33} N_{1}^{257} N_{2}^{217}}
$$

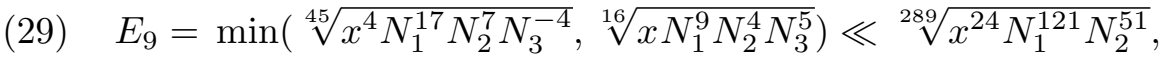

(30) $E_{10}=\min \left(\sqrt[16]{x N_{1}^{9} N_{2}^{10} N_{3}^{-1}}, \sqrt[16]{x N_{1}^{9} N_{2}^{4} N_{3}^{5}}\right) \ll \sqrt[96]{x^{6}\left(N_{1} N_{2}\right)^{54}}$. 
By (21) and (22) we get

(31) $x^{-\eta} S(a, b, c, d ; \boldsymbol{N}) \ll \min \left(\sqrt[16]{x N_{1}^{9} N_{2}^{4} N_{3}^{5}}, \sqrt[8]{x N_{1}^{-3} N_{2}^{-2} N_{3}^{-1}}\right)+x^{0.1}$

$$
\ll \sqrt[28]{\left(x N_{1}^{-1} N_{2}^{-1}\right)^{3}}+x^{0.1}
$$

For brevity we set $J=N_{1} N_{2}$. From (23) to (31) we find that

$$
x^{-\eta} S(a, b, c, d ; \boldsymbol{N}) \ll \sum_{11 \leq i \leq 17} E_{i}+x^{0.1},
$$

where

$$
\begin{aligned}
& E_{11}=\min \left(\sqrt[28]{\left(x J^{-1}\right)^{3}}, \sqrt[33]{x^{3} J^{7}}\right) \ll x^{6 / 59}, \\
& E_{12}=\min \left(\sqrt[28]{\left(x J^{-1}\right)^{3}}, \sqrt[888]{x^{73} J^{269.5}}\right) \ll x^{0.1007}, \\
& E_{13}=\min \left(\sqrt[28]{\left(x J^{-1}\right)^{3}}, \sqrt[76]{x^{6} J^{26.5}}\right) \ll x^{0.1006}, \\
& E_{14}=\min \left(\sqrt[28]{\left(x J^{-1}\right)^{3}}, \sqrt[116]{x^{6} J^{79}}\right) \ll x^{0.1} \\
& E_{15}=\min \left(\sqrt[28]{\left(x J^{-1}\right)^{3}}, \sqrt[488]{x^{33} J^{237}}\right) \ll x^{0.1} \\
& E_{16}=\min \left(\sqrt[28]{\left(x J^{-1}\right)^{3}}, \sqrt[289]{x^{24} J^{86}}\right) \ll x^{0.1008} \\
& E_{17}=\min \left(\sqrt[28]{\left(x J^{-1}\right)^{3}}, \sqrt[48]{x^{3} J^{27}}\right) \ll x^{0.1}
\end{aligned}
$$

as required.

\section{References}

[1] A. Baker, A Concise Introduction to the Theory of Numbers, Cambridge Univ. Press, 1984.

[2] P. T. Bateman and E. Grosswald, On a theorem of Erdös and Szekeres, Illinois J. Math. 2 (1958), 88-98.

[3] P. Erdős and G. Szekeres, Über die Anzahl der Abelschen Gruppen gegebener Ordnung und über ein verwandtes zahlentheoretisches Problem, Acta Sci. Math. (Szeged) 7 (1935), 95-102.

[4] A. Ivić, On the asymptotic formulas for powerful numbers, Publ. Inst. Math. (Belgrade) 23 (37) (1978), 85-94.

[5] - On the number of finite non-isomorphic abelian groups in short intervals, Math. Nachr. 101 (1981), 257-271.

[6] A. Ivić and P. Shiu, The distribution of powerful numbers, Illinois J. Math. 26 (1982), 576-590.

[7] E. Krätzel, Zahlen k-ter Art, Amer. J. Math. 44 (1972), 309-328.

[8] —, Divisor problems and powerful numbers, Math. Nachr. 114 (1983), 97-104.

[9] - Z Zweifache Exponentialsummen und dreidimensionale Gitterpunktprobleme, in: Elementary and Analytic Theory of Numbers, Banach Center Publ. 17, PWN, Warszawa, 1985, 337-369.

[10] - , The distribution of powerful integers of type 4, Acta Arith. 52 (1989), 141-145. 
[11] H.-Q. Liu, On the number of abelian groups of a given order, ibid. 59 (1991), 261-277.

[12] - On the number of abelian groups of a given order (supplement), ibid. 64 (1993), 285-296.

[13] - , The greatest prime factor of the integers in an interval, ibid. 65 (1993), 301-328.

206-10, BAO GUO ST.

HARBIN, 150066

CHINA

Received on 26.7.1993

and in revised form on 9.2.1994 